\title{
Panorama do Uso de Métodos Quantitativos em Pesquisas sobre Marketing de Relacionamento com Egressos.
}

\author{
Glauco José Ribeiro Borges (UNISUL) - glaucojrb@gmail.com \\ Sandro Vieira Soares (UNISUL) - sandrovieirasoares@hotmail.com \\ Carlos Rogério Montenegro de Lima (UNISUL) - carlos.montenegro@unisul.br \\ Aléssio Bessa Sarquis (UNISUL) - alessio.sarquis@gmail.com \\ Isabella Ramos Boing (UNISUL) - bellars@gmail.com
}

\begin{abstract}
RESUMO
O uso adequado de métodos quantitativos tem se mostrado como ponto crítico no desenvolvimento de pesquisas quantitativas na área de Administração e o desenvolvimento científico da área de estatística somada ao crescimento da capacidade computacional e o surgimento ou melhoramento dos softwares disponíveis no mercado são responsáveis pelo oferecimento de uma ampla variedade opções metodológicas quantitativas aos pesquisadores da área. $\mathrm{O}$ estudo buscou descrever o panorama dos métodos quantitativos empregados em pesquisas sobre marketing de relacionamento com egressos. Trata-se de um estudo bibliográfico que analisou um portfólio bibliográfico de 20 artigos publicados até 2018 sobre a temática. Os resultados mostram que a maior parte das publicações na temática utilizam as técnicas modelagem de equações estruturais, análise fatorial confirmatória e técnicas de estatística descritiva. O Alfa de Cronbach é a técnica de análise de confiabilidade de escalas mais utilizada. As duas variáveis mais analisadas nos estudos na temática são imagem da universidade e satisfação, termos amparados pela literatura da área. O estudo contribuiu para ampliar a compreensão sobre o uso de métodos quantitativos em pesquisas na temática e apontar alguns direcionamentos que podem auxiliar em futuros estudos.
\end{abstract}

PALAVRAS-CHAVE: Métodos quantitativos; Técnicas estatísticas; Marketing de relacionamento; Egressos.

\section{Overview of the Use of Quantitative Methods in Research on Relationship Marketing with Alumni.}

\begin{abstract}
The appropriate use of quantitative methods is a critical point in the development of quantitative research in the Business area and the scientific development of the statistics area, in addition to the growth of computational capacity and emergence or improvement of software available on the market are responsible for offering a wide variety of quantitative methodological options to researchers in the field. The study maps an overview of the quantitative methods used in research on relationship marketing with alumni. It is a bibliographic study which analyzed a bibliographic portfolio of 20 papers published until 2018 on the subject. The results show that most publications on the subject use structural equation modeling, confirmatory factor analysis and descriptive statistics. Cronbach's alpha is the most used scale reliability analysis. The two variables most analyzed in studies on the subject are university image and satisfaction, terms supported by the literature in the area. The study contributed to broaden the understanding about the use of quantitative methods in research on the subject and to point some directions that may help in future studies.
\end{abstract}

KEYWORDS: Quantitative methods; Statistics; Marketing of relationship; Alumni.

\section{vff \\ Fluminense}

R. Desembargador Ellis Hermydio Figueira, 783, Bloco A, sl. 218, Aterrado. 27213-415 - Volta Redonda, RJ - Brasil

www.uff.br

Copyright (C) 2020 RASI. Todos os direitos, até mesmo de tradução, são reservados. É permitido citar parte de artigos sem autorização prévia, desde que seja identificada a fonte. 


\section{Panorama do Uso de Métodos Quantitativos em Pesquisas sobre Marketing de Relacionamento com Egressos.}

\section{Introdução}

$\mathrm{Na}$ área de Administração, a literatura de metodologia de pesquisa apresenta três grandes abordagens de análise de dados: quantitativa, qualitativa e mista. Uma série de pesquisas no Brasil utilizaram métodos quantitativos, ou seja, técnicas de estatística na análise dos dados, como os trabalhos de Dallabona, Nascimento e Hein (2010), Gouvêa, Prearo e Romeiro (2010), Gouvêa, Prearo e Romeiro (2011), Gouvêa, Prearo e Romeiro (2012a), Gouvêa, Prearo e Romeiro (2012b), Gouvêa, Prearo e Romeiro (2013), Hosser, Cruz e Quintana (2018), Prearo, Gouvêa, Monari e Romeiro (2011), Prearo, Gouvêa e Romeiro (2011b), Prearo, Gouvêa e Romeiro (2012) e Prearo, Gouvêa e Romeiro (2011a). Esses estudos utilizaram estudos utilizaram diferentes técnicas, procedimentos e a verificação de alguns pressupostos estatísticos, já que isso é um ponto crítico nos estudos de abordagem quantitativa.

Discutir o desenvolvimento de pesquisas com abordagem quantitativas é importante, dado que autores como Ferreira e Falaster (2016) apontaram que amostras enviesadas, pouco representativas, com detalhamento deficiente das variáveis empregadas ou com inadequação na forma de tratamento dos dados coletados são algumas das principais causas de rejeição de artigos científicos em revistas brasileiras, e que a seção ligada ao método é aquela que mais contribui para a rejeição direta de artigos (desk rejection). Assim, a adequação metodológica tem sido um critério relevante utilizado por pareceristas de eventos e periódicos científicos na área de Administração e Contabilidade (Espejo, Azevedo, Trombelli, \& Voese, 2013). Questões relacionadas ao método, como falta de clareza no processo de amostragem e técnica estatística inadequada, são aspectos que mais contribuem para a reprovação de artigos na Revista Brasileira de Contabilidade (Almeida, 2014).

Para além dos efeitos durante o processo de peer review de escolhas metodológicas inadequadas em pesquisas quantitativas há ainda a constatação da pesquisa deFiates, Serra e Martins (2014) acerca do uso de técnicas estatísticas por pesquisadores brasileiros que chegou a duas conclusões. A primeira é que os pesquisadores brasileiros têm proficiência apenas em técnicas simples de análise como correlação e teste $t$, carecendo de domínio em técnicas mais sofisticadas. Segundo que os pesquisadores brasileiros se sentem menos preparados que os norte-americanos no uso de técnicas estatísticas de análise de dados. Prearo et al. (2011) concluíram também que a maioria das pesquisas brasileiras na área não faz a verificação adequada dos pressupostos necessários das técnicas de estatística empregadas.

Com efeito, a escolha da técnica estatística adequada não é um processo trivial. Lana, Partyka, Alberton e Marcon (2018) afirmaram que existem razões pelas quais técnicas estatísticas específicas são mais utilizadas que outras. Uma dessas razões pode ser a menor complexidade de aplicação de algumas técnicas na análise de dados. Soares, Picolli e Casagrande (2018) afirmaram também que as pesquisas bibliográficas podem auxiliar os futuros pesquisadores na escolha da técnica de estatística necessária, conforme o propósito do estudo.

Diante deste contexto, apresenta-se a seguinte questão de pesquisa: Quais métodos quantitativos são empregados em pesquisas sobre marketing de relacionamento com egressos? 
Com isso, este estudo visa descrever o panorama do uso de métodos quantitativos nas pesquisas sobre marketing de relacionamento com egressos.

Lordelo e Dazanni (2012) constataram que a despeito da crescente percepção de que as instituições de ensino vêm desenvolvendo cada vez mais esforços para construir relacionamentos com seus estudantes e egressos, acredita-se que há ainda poucos estudos empíricos sobre esta temática, com abordagem quantitativa e uso de técnicas de estatística na análise dos dados, o que justifica o desenvolvimento da presente pesquisa.

\section{Fundamentação Teórica}

Nesta seção, apresenta-se uma breve abordagem acerca de algumas técnicas estatísticas comumente utilizadas em estudos sobre marketing de relacionamento com egressos, seguida de uma síntese dos achados sobre o emprego de métodos quantitativos na análise de dados nos estudos sobre a temática.

\subsection{Métodos quantitativos de análise de dados}

A pesquisa científica se vale de dados coletados para obter conclusões relevantes, geralmente embasadas em respostas a questionamentos propostos. Conforme Barbetta (2017), para que tais resultados sejam confiáveis, tanto a coleta de dados quanto a análise de dados precisariam ser criteriosamente realizadas. Assim, nas pesquisas quantitativas sobre o tema marketing de relacionamento com egressos há o uso de diversos métodos ou técnicas estatísticas e análise, que serão apresentados na sequência.

Dentre os métodos estatísticos quantitativos utilizados estão os métodos univariados, quais sejam: as técnicas de estatística descritiva, os testes de hipótese paramétricos e a análise de variância (ANOVA). No que tange à Estatística descritiva, conforme Larson e Farber (2010), esta pode ser tomada como uma vertente estatística que aborda a organização, o resumo e a representação dos dados. Por meio dessas técnicas é possível descrever e elencar as principais características verificadas em um determinado conjunto de dados, por meio de tabelas, gráficos e medidas-resumo, sem apresentar quaisquer conclusões ou inferências acerca da população (Fávero, \& Belfiore, 2017).

Field (2009) enumerou como principais medidas descritivas as seguintes: medidas de tendência central (mediana e moda), medidas de dispersão (variância e desvio padrão) e as medidas de assimetria e curtose. Em geral, as técnicas de estatística descritiva possuem o propósito de organizar, resumir e apresentar os dados coletados, facilitando a sua interpretação, atendendo aos objetivos do estudo (Barbetta, 2017).

Em relação aos testes paramétricos, quando é possível conhecer a distribuição de probabilidades teórica da variável em estudo, pode-se estimar os parâmetros e realizar, de forma otimizada, testes de hipóteses sobre relação entre variáveis. Conforme Chen e Popovich (2002) e Malhotra (2012), os testes paramétricos incluem o requisito de que a variável em análise tenha distribuição de probabilidade conhecida, supondo que a variável analisada tenha sido medida no mínimo em escala intervalar; em alguns casos, há também a necessidade de as variáveis mensuradas terem variâncias homogêneas, ou seja, homocedasticidade.

Cooper e Schindler (2016) afirmaram que os testes de hipóteses paramétricos são mais pertinentes, em relação aos não paramétricos, em virtude dos seus dados analisados serem derivados de mensuração em escala métrica. Além disso, segundo esses autores, esses testes requerem uma série de pressupostos para serem utilizados, quais sejam: as observações devem ser independentes; as observações devem ser retiradas de populações normalmente distribuídas; essas populações devem ter variâncias iguais; as escalas de 
mensuração devem estar no menor intervalo para que se possam efetuar operações aritméticas com as mesmas; uma vez que os testes de hipóteses paramétricos são uma técnica de inferência estatística, a amostra em estudo deve ser probabilística, caso contrário não seria possível a extrapolação dos resultados para a população-alvo do estudo.

Sobre a análise de variância, também conhecida como ANOVA, Hair Junior, Black, Babin, Anderson, e Tatham, (2009) asseveraram que é uma técnica estatística indicada quando o objetivo do estudo é determinar se as amostras de dois ou mais grupos surgem de populações com médias iguais, ou seja, se permitem testar hipóteses sobre médias de distintas populações. Segundo esses autores, assim como outros testes de hipóteses, a análise de variância se estrutura sob algumas suposições (ou pressupostos) para ser aplicável, quais sejam: todas as observações devem ser independentes; as observações em cada grupo devem possuir uma distribuição aproximadamente normal; as populações devem ter variâncias iguais, ou seja, homoscedasticidade. De acordo com Levin, Fox e Forde (2012), além dessas premissas, outra condição é dispor de dados que resultaram de escalas intervalares.

Nos estudos sobre o tema há, também, a presença de outros métodos quantitativos de análise, classificados por Hair Junior et al. (2009) como métodos de estatística multivariada. Tais métodos compreendem um conjunto de técnicas que objetivam analisar dados que possuem a característica de expansão e que incluam um vasto domínio de possíveis situações de pesquisa. Eles proporcionam uma análise simultânea de múltiplas variáveis em um único conjunto de relações, auxiliando na compreensão de comportamentos complexos e permitindo preservar as correlações naturais entre as múltiplas influências de comportamento, sem isolar qualquer indivíduo ou variável. Com isso, conforme apontado por Steiner (1995), a frequente necessidade dos pesquisadores de entender a relação entre diversas variáveis aleatórias faz da análise multivariada uma metodologia com grande potencial de uso. As técnicas multivariadas mais utilizadas nos estudos sobre marketing de relacionamento com egressossão: análise de cluster (agrupamentos), análise discriminante, análise de regressão múltipla, análise de regressão logística, modelagem de equações estruturais (SEM), análise fatorial exploratória, análise fatorial confirmatória e análise de correlação.

Sobre a análise de cluster (ou agrupamentos), sabe-se que é comum aos pesquisadores encontrarem situações que requerem o agrupamento de objetos homogêneos (Hair Junior et al., 2009). Nessas situações, a técnica de análise mais utilizada é a análise de cluster, cuja finalidade é agrupar objetos segundo suas características, formando grupos ou conglomerados homogêneos. Sobre essa técnica, Roses e Leis (2002) chamam a atenção para que os conglomerados obtidos devam apresentar tanto homogeneidade interna (dentro de cada conglomerado), como heterogeneidade externa (entre os conglomerados). Se a aglomeração for bem-sucedida, os objetos dentro dos conglomerados estarão muito próximos; enquanto nos conglomerados distintos os objetos estarão afastados.

A análise de cluster é uma técnica do tipo interdependência, já que não permite determinar antecipadamente as variáveis dependentes e independentes em análise (Roses, \& Leis, 2002). Ao contrário, ela permite examinar relações de interdependência entre todo o conjunto de variáveis. Como o seu objetivo é agrupar objetos semelhantes, essa técnica requer uma medida da distância entre os objetos analisados. Os objetos com menor distância entre si são mais semelhantes, logo se encontram em um mesmo conglomerado; ao passo que os objetos mais distantes participam de conglomerados distintos. Portanto, o conceito de 
similaridade é fundamental na análise de clusters, podendo ser aferida por medidas de correlação, medidas de distância e medidas de associação.

Com relação à análise discriminante, também conhecida como análise do fator discriminante (ou análise discriminante canônica), Prearo et al. (2010) relataram que embora esta técnica tenha sido originalmente desenvolvida para a área de Botânica, logo se expandiu para outras áreas, como gestão e marketing, em função de suas possibilidades de encontrar grupos de indivíduos e características que os distinguem uns dos outros. A análise discriminante se enquadra na categoria de investigação de dependência entre as variáveis analisadas e como uma técnica de análise preditiva. Gouvêa et al. (2012) apresentam uma relação de premissas teóricas que precisam ser observadas na aplicação da técnica análise discriminante, que são inerentes ao processo de construção teórica da técnica. Elas garantem que o algoritmo da técnica tenha o comportamento que se espera dele. Segundo esses autores, tais premissas são: baixa multicolinearidade dos dados, ausência de observações atípicas, homoscedasticidade, linearidade, normalidade multivariada, tamanho da amostra e tamanho dos grupos analisados.

Já as técnicas de regressão, conforme Fávero, Belfiori, Silva, \& Chan (2009), analisam o comportamento de uma variável dependente em função de uma ou mais variáveis explicativas, e possibilitam o desenvolvimento de modelos preditivos. Sobre a análise de regressão múltipla, Hair Junior et al. (2009) relatam que, diferentemente dos casos em que o problema envolve apenas uma variável independente, onde a técnica indicada é a análise de regressão simples, essa é uma técnica utilizada para analisar a relação entre uma única variável dependente e várias variáveis independentes, geralmente com propósito de prever valores da variável dependente. Assim, os modelos de regressão múltipla apresentam duas os mais variáveis explicativas, enquanto os modelos de regressão simples apresentam apenas uma única variável. Prearo et al. (2011a) apontaram como pressupostos dessa técnica os seguintes: ausência de multicolinearidade, ausência de dados atípicos, tamanho da amostra, homoscedasticidade, normalidade multivariada, linearidade dos dados e a ausência de erros correlacionados.

Ao passo que a análise de regressão logística é uma técnica que tem como objetivo produzir, a partir de um conjunto de observações, um modelo que permita a predição de valores tomados por uma variável categórica, frequentemente binária, a partir de uma série de variáveis explicativas contínuas ou binárias (Agresti, 2019). A análise de regressão logística (também conhecida como modelo logístico, modelo logit, ou classificador de máxima entropia) é o modelo mais popular na análise de dados binários, sendo amplamente usada tanto em ciências médicas como nas ciências sociais.

Sobre a aplicação da regressão logística, Prearo et al. (2009) recomendam tanto na categoria de investigação da dependência entre as variáveis quanto na categoria de predição. Eles asseveram ainda que, sob a ótica das premissas, que geralmente permeiam a utilização de técnicas de dependência, que sejam observados a normalidade dos dados, a multivariada e a homoscedasticidade. O grande mérito desse método de estimação reside em apresentar boas propriedades assintóticas dos estimadores, que são consistentes e assintoticamente eficientes, requerendo o atendimento de bem menos pressupostos que outras técnicas afins, como a análise discriminante ou a regressão múltipla. Sendo assim, os pressupostos presentes na análise de regressão logística são: baixa multicolinearidade, ausência de observações atípicas e tamanho da amostra.

Com relação à modelagem de equações estruturais - também compreendida como modelagem causal, análise causal, modelagem por equações simultâneas, análise de estruturas de covariância e LISREL - compreende um grupo de modelos estatísticos que buscam 
explicar as relações entre múltiplas variáveis, e é uma extensão de técnicas multivariadas, mais especificamente, a análise de regressão múltipla e análise fatorial (Hair Junior et al., 2009). A técnica de modelagem de equações estruturais é considerada uma evolução da modelagem de multiequações, desenvolvida principalmente na área de Econometria, e também dos princípios de mensuração da Psicologia e Sociologia. Prearo et al. (2011b) apontaram que a sua aplicação é bastante recente nos trabalhos das Ciências Humanas Aplicadas, particularmente na área da Administração.

A técnica de modelagem de equações estruturais é comumente aplicada na construção de hipóteses e testes, e seus principais objetivos são (Prearo, Gouvêa, \& Romeiro, 2011b): testar teorias a partir de hipóteses, avaliar a significância estatística de um modelo teórico e especificar relações causais entre variáveis latentes. De acordo com Hair Junior et al. (2009), a modelagem de equações estruturais é definida por três características distintas: estimação de relações de dependência múltiplas e inter-relacionadas; habilidade para representar conceitos não observados nessas relações e corrigir erro de mensuração no processo de estimação; definição de um modelo para explicar o conjunto inteiro de relações. Assim, conforme Prearo et al. (2011b), os pressupostos subjacentes ao uso dessa técnica são: normalidade, multicolinearidade moderada, ausência de observações atípicas, linearidade dos dados e o tamanho da amostra.

Por fim, outro método identificado nos estudos sobre marketing de relacionamento com egressos é a análise fatorial. Hair Junior et al. (2009) a consideram como uma técnica multivariada fundamental, pois desempenha um importante papel na aplicação de outras técnicas multivariadas, podendo ser utilizada tanto na perspectiva exploratória como confirmatória. No entanto, embora tenham semelhanças, a análise fatorial exploratória e a análise fatorial confirmatória são técnicas distintas. A análise fatorial exploratória fornece informações sobre quantos fatores são necessários para melhor representar os dados, e todas as variáveis medidas são relacionadas com cada fator por uma estimativa de carga fatorial (Hair Junior et al., 2009). Já na análise fatorial confirmatória deve-se especificar o número de fatores que existem dentro de um conjunto de variáveis e sobre qual fator cada variável irá carregar antes que os resultados sejam computados (Hair Junior et al., 2009). Portanto, como destacado por Lattin, Carroll e Green (2011), na análise fatorial exploratória se objetiva inferir a estrutura fatorial mediante padrões de correlação nos dados; ao passo que na análise fatorial confirmatória se tem uma noção prévia da estrutura do modelo fatorial, sendo que tal noção preliminar é forte o suficiente para determinar o modelo.

Por fim, faz-se adequado ainda apresentar nesta seção os trabalhos cujo enfoque foi a utilização de métodos quantitativos na realização de pesquisa científica, a fim de elencar seus principais achados e conclusões, e principalmente em relação às técnicas estatísticas utilizadas. Além dos estudos de Gouvêa et al. (2010; 2011; 2012a; 2013), Prearo et al. (2011), Prearo et al. (2009; 2010) e Prearo et al. (2011a; 2011b; 2012), que já foram abordados na seção anterior, outros estudos que compõem o portfólio bibliográfico desta pesquisa são os trabalhos de Dallabona et al. (2010), Silva, Wanderley e Santos (2010) e Hosser et al. (2018), cujos principais achados estão apresentados a seguir.

Dallabona et al. (2010) mapearam dissertações de um programa de pós-graduação em Contabilidade, no período de 2005 a 2009, e analisaram os métodos estatísticos de análise aplicados. Seus resultados indicam a predominância do emprego de técnicas de estatística descritiva $(50 \%)$, e o uso de outras técnicas de inferência estatística, como regressão e correlação, análise multivariada de dados, abordagem multicritério, modelagem matemática, metaheurística e data mining. Esses autores afirmam que ocorreu uma evolução, nos anos 
recentes, no emprego de métodos de análise multivariada, principalmente técnicas de regressão e correlação.

Silva et al. (2010) analisaram 299 artigos apresentados no Congresso ANPCONT, no Congresso USP de Controladoria e Contabilidade e no EnANPAD entre os anos de 2007 e 2009, buscando identificar o grau de aderência da metodologia quantitativa à análise de dados. Seus resultados apontam que mais de $50 \%$ dos artigos utilizaram abordagem estatística na análise dos dados, com o emprego de 11 diferentes tipos de técnicas: técnicas de inferência estatística, análise de regressão e técnicas de estatística descritiva. Esses autores constataram que a quantidade de artigos com metodologia quantitativa vem aumentando no decorrer dos anos, subindo de 46\% em 2007 para 62\% em 2009, e que a metodologia está em 162 trabalhos da amostra analisada.

Já Hosser et al. (2018), que mapearam 637 trabalhos publicados em nove edições do congresso promovido pela ANPCONT, identificaram o uso de 30 técnicas estatísticas consideradas como principais, 14 como técnicas categorizadas como autônomas, 148 como técnicas acessórias e 6 técnicas identificadas como "outros grupos". As conclusões desse estudo são que os trabalhos analisados possuem características diferentes quanto ao emprego de técnicas estatísticas, e que os pesquisadores da área utilizam uma ampla gama de técnicas estatísticas na análise dos dados.

\section{Aspectos Metodológicos}

Esta seção aborda delineamento e procedimentos metodológicos utilizados nesta pesquisa. Quanto ao objetivo, o presente estudo pode ser classificado como descritivo, conforme Cooper e Schindler (2016), que assim define os estudos que buscam descrever (ou definir um assunto), criando o perfil de um grupo de problemas, pessoas ou eventos. Hair Junior, Babin, Money e Samouel (2005) assim define o estudo que se estrutura de modo a mensurar as características descritas na questão de pesquisa, utilizando técnicas padronizadas na coleta de dados. Quanto à abordagem, este estudo é predominantemente quantitativo, uma vez que busca coletar dados quantitativos sobre ocorrências fragmenta (e delimita) o fenômeno analisado em categorias mensuráveis, e busca estabelecer padrões (Gray, 2012; Sampieri, Collado, \& Lúcio, 2013). Cooper e Schindler (2016) afirmaram que predominam na pesquisa quantitativa o uso de métodos estatísticos e matemáticos de análise, e que este tipo de pesquisa envolve uma forma de investigação sequencial, em que as etapas precedem umas às outras.

\subsection{Seleção do Portfólio Bibliográfico a ser Analisado}

O levantamento dos estudos que compõem a amostra desta pesquisa teve início com a definição do período de publicações a serem investigadas, o qual foi inicialmente limitado aos últimos dez anos, buscando captar as pesquisas mais recentes. Entretanto, em virtude do número reduzido de publicações identificadas no intervalo definido, optou-se por não se estipular o ano inicial das publicações, mas coletar estudos publicados até 2018. Em seguida, houve a definiçãa das bases de dados a serem consultadas. Em virtude da relevância para as atividades de ensino e pesquisa em nível de pós-graduação, as seguintes bases de dados foram selecionadas: EBSCO, Redalyc, Portal de periódicos CAPES/MEC, Scopus, Spell, Web of Science e Google Acadêmico.

Uma vez definidas as bases de dados, coletou-se os artigos publicados em periódicos científicos (não em Anais de eventos, livros, dissertações ou teses) e disponibilizados em formato de texto completo. Além disso, utilizou-se como descritores de busca os seguintes: 
"relationship marketing"; "alumni", "marketing de relaciones", "egressado"; "marketing de relacionamento"; "relacionamento" e "egresso", tanto no singular como no plural.

O processamento e filtragem do portfólio bibliográfico inicial obtidos (que contou com 273 artigos)se deu em 3 etapas eliminatórias e consecutivas. A primeira filtragem envolveu o descarte dos artigos repetidos ( 80 trabalhos) e aqueles não publicados em revistas científicas (149 trabalhos). Posteriormente, fez-se uma análise detalhada do resumo e das palavras-chaves dos 44 artigos selecionados, e foram excluídos mais 24 trabalhos, que não estavam plenamente alinhados com o tema e objetivo desta pesquisa - o que inclui os artigos que utilizaram somente métodos de análise qualitativos. Assim, o resultando desse processamento revelou um portfólio bibliográfico final com 20 artigos, apresentado no Quadro 01 da próxima seção do artigo. Embora o tema marketing de relacionamento possua um grande volume de publicações na literatura, o portfólio bibliográfico final aqui selecionado contém somente 20 artigos científicos. Supostamente, em razão do propósito deste estudo, qual seja, analisar somente os estudos sobre marketing de relacionamento com egressos e com abordagem quantitativa na análise dos dados.

\section{Apresentação e Análise dos Resultados}

\subsection{Caracterização da Amostra}

Após os procedimentos de busca e seleção de artigos descritos na seção 3.1, construiuse uma amostra de artigos cujos títulos, objetivos, autores e ano de publicação são apresentados no Quadro 1 a seguir.

Quadro 1 - Artigos da amostra da pesquisa

\begin{tabular}{|c|c|c|c|}
\hline Título do Artigo & Objetivo do Artigo & Autores & Ano \\
\hline $\begin{array}{l}\text { The Relationship } \\
\text { between Alumni and } \\
\text { University: Toward a } \\
\text { Theory of } \\
\text { Discretionary } \\
\text { Collaborative } \\
\text { Behavior }\end{array}$ & $\begin{array}{c}\text { Explorar os antecedentes do comportamento colaborativo } \\
\text { discricionário realizado por ex-alunos da universidade a } \\
\text { fim de auxiliar sua alma mater. }\end{array}$ & $\begin{array}{l}\text { Heckman; } \\
\text { Guskey. }\end{array}$ & 1998 \\
\hline $\begin{array}{l}\text { Student Trust and Its } \\
\text { Antecedents in } \\
\text { Higher Education }\end{array}$ & $\begin{array}{c}\text { Sugerir uma abordagem alternativa de longo prazo para } \\
\text { controlar os custos de marketing construindo confiança } \\
\text { dos alunos em sua faculdade. }\end{array}$ & $\begin{array}{l}\text { Ghosh; } \\
\text { Whipple; } \\
\text { Bryan. }\end{array}$ & 2001 \\
\hline $\begin{array}{l}\text { University } \\
\text { Experiences, the } \\
\text { Student-College } \\
\text { Relationship, and } \\
\text { Alumni Support }\end{array}$ & $\begin{array}{c}\text { O objetivo desta pesquisa é desenvolver percepções } \\
\text { práticas que possam oferecer aos profissionais de } \\
\text { marketing da universidade uma estrutura a partir da qual } \\
\text { avaliem vários programas (por exemplo, atividades no } \\
\text { campus ou eventos de ex-alunos) por sua contribuição } \\
\text { pote. }\end{array}$ & $\begin{array}{l}\text { McAlexander; } \\
\text { Koenig. }\end{array}$ & 2001 \\
\hline $\begin{array}{l}\text { Experiential Bases } \\
\text { for Relationship } \\
\text { Development: A } \\
\text { Study of Alumni } \\
\text { Relationships. } \\
\end{array}$ & $\begin{array}{l}\text { O objetivo principal desta pesquisa foi entender as } \\
\text { emoções evocadas pela participação em uma cerimônia de } \\
\text { graduação que marcou a conclusão do envolvimento da } \\
\text { maioria dos estudantes com a universidade. }\end{array}$ & $\begin{array}{c}\text { Palmer; } \\
\text { Koenig-Lewis. }\end{array}$ & 2008 \\
\hline $\begin{array}{l}\text { Managing students' } \\
\text { loyalty to school after } \\
\text { graduation through } \\
\text { relationship } \\
\text { marketing }\end{array}$ & $\begin{array}{l}\text { O objetivo do estudo é considerar a expectativa do cliente, } \\
\text { o desempenho do serviço percebido e a satisfação do } \\
\text { cliente como questões de Gestão da Qualidade Total no } \\
\text { marketing de relacionamento, e examina como eles se } \\
\text { relacionam à lealdade antecipada dos alunos à faculdade } \\
\text { após a graduação. }\end{array}$ & $\begin{array}{l}\text { Ehigie; } \\
\text { Taylor. }\end{array}$ & 2009 \\
\hline
\end{tabular}




\begin{tabular}{|c|c|c|c|}
\hline \multicolumn{4}{|c|}{ Quadro 1 - Artigos da amostra da pesquisa (continuação) } \\
\hline Título do Artigo & Objetivo do Artigo & Autores & Ano \\
\hline $\begin{array}{l}\text { Validação de um modelo } \\
\text { de lealdade do estudante } \\
\text { com base na qualidade do } \\
\text { relacionamento. }\end{array}$ & $\begin{array}{l}\text { Verificar as relações existentes entre qualidade } \\
\text { percebida, comprometimento, confiança e lealdade, } \\
\text { no contexto do serviço educacional, utilizando o } \\
\text { modelo de lealdade do estudante com base na } \\
\text { qualidade do relacionamento, proposto por Hennig- } \\
\text { Thurau, Langer e Hansen (2001). }\end{array}$ & $\begin{array}{l}\text { Torres; } \\
\text { Sittoni. }\end{array}$ & 2009 \\
\hline $\begin{array}{l}\text { La identificación egresado- } \\
\text { universidad: un modelo } \\
\text { desde un enfoque de } \\
\text { marketing relacional. }\end{array}$ & $\begin{array}{c}\text { Propor um modelo de relações em torno da estrutura } \\
\text { da aprendizagem ao longo da vida ou da formação ao } \\
\text { longo da vida do indivíduo no ensino superior e suas } \\
\text { consequiências sobre o desempenho da universidade, } \\
\text { no nível da relação pós-graduação. }\end{array}$ & $\begin{array}{l}\text { Schlesinger; } \\
\text { Cervera; } \\
\text { Iniesta. }\end{array}$ & 2012 \\
\hline $\begin{array}{l}\text { Medición de la imagen de } \\
\text { la universidad y sus } \\
\text { efectos sobre la } \\
\text { identificación y lealtad del } \\
\text { egresado: una } \\
\text { aproximación desde el } \\
\text { modelo de Beerli y Díaz } \\
\text { (2003). }\end{array}$ & $\begin{array}{l}\text { Desenvolver um modelo para medir a imagem da } \\
\text { Universidade percebida por seus graduados e analisar } \\
\text { a influência que essa imagem exerce na identificação } \\
\text { e lealdade do graduado à sua instituição. }\end{array}$ & $\begin{array}{l}\text { Cervera; } \\
\text { Schlesinger; } \\
\text { Mesta; } \\
\text { Sánchez. }\end{array}$ & 2012 \\
\hline $\begin{array}{l}\text { Hail to Thee, Our Alma } \\
\text { Mater: Alumni Role } \\
\text { Identity and the } \\
\text { Relationship to } \\
\text { Institutional Support } \\
\text { Behaviors. } \\
\end{array}$ & $\begin{array}{l}\text { Avaliar como os egressos de faculdades e } \\
\text { universidades vêem seu papel nessas instituições } \\
\text { após a graduação, e como essa percepção se relaciona } \\
\text { com os comportamentos de apoio. }\end{array}$ & McDearmon. & 2013 \\
\hline $\begin{array}{l}\text { El papel de la confianza, la } \\
\text { imagen y los valores } \\
\text { compartidos en la creación } \\
\text { de valor y lealtad: } \\
\text { Aplicación a } \\
\text { larelaciónegresado- } \\
\text { universidad. }\end{array}$ & $\begin{array}{c}\text { O estudo objetiva analisar o processo de intercâmbio } \\
\text { relacional entre instituições de ensino superior e seus } \\
\text { graduados, a fim de propor a aplicação do marketing } \\
\text { de relacionamento no campo do ensino superior, } \\
\text { especificamente no relacionamento egresso- } \\
\text { universidade. }\end{array}$ & $\begin{array}{l}\text { Schlesinger; } \\
\text { Cervera; } \\
\text { Calderón. }\end{array}$ & 2014 \\
\hline $\begin{array}{l}\text { The Identity Salience and } \\
\text { Emotional Attachment } \\
\text { Strategies in Alumni- } \\
\text { University Relationships. }\end{array}$ & $\begin{array}{l}\text { Abordar a questão do marketing de relacionamento } \\
\text { de longo prazo. }\end{array}$ & $\begin{array}{l}\text { Min; Segal; } \\
\text { Dalman. }\end{array}$ & 2014 \\
\hline $\begin{array}{l}\text { Un enfoque de marketing } \\
\text { de relaciones a la } \\
\text { educación como un } \\
\text { servicio: A aplicación a la } \\
\text { Universidad de Valencia. }\end{array}$ & $\begin{array}{c}\text { Aplicar o paradigma do marketing de relacionamento } \\
\text { à análise da gestão educacional. Mais } \\
\text { especificamente, o que se busca é construir um } \\
\text { modelo integrado de relações entre o egresso e a } \\
\text { universidade. }\end{array}$ & $\begin{array}{l}\text { Schlesinger; } \\
\text { Cervera; } \\
\text { Iniesta; } \\
\text { Sánchez. }\end{array}$ & 2014 \\
\hline $\begin{array}{l}\text { Key Elements in Building } \\
\text { Relationships in the } \\
\text { Higher Education Services } \\
\text { Context }\end{array}$ & $\begin{array}{l}\text { Redefinir a lacuna entre serviços educacionais e } \\
\text { marketing de relacionamento na literatura, } \\
\text { explorando as percepções de ex-alunos em relação à } \\
\text { experiência e serviço em termos de uma abordagem } \\
\text { de aprendizagem e retenção ao longo da vida. }\end{array}$ & $\begin{array}{l}\text { Schlesinger; } \\
\text { Cervera; } \\
\text { Iniesta; } \\
\text { Sánchez. }\end{array}$ & 2015 \\
\hline $\begin{array}{l}\text { Dimensões do } \\
\text { relacionamento entre a } \\
\text { universidade e seus } \\
\text { egressos por meio da } \\
\text { informação caso da UFMG }\end{array}$ & $\begin{array}{l}\text { Analisar o relacionamento entre uma IES e seus ex- } \\
\text { alunos, no caso, a Universidade Federal de Minas } \\
\text { Gerais (UFMG), representada pelo seu programa de } \\
\text { egressos, o Sempre UFMG, concebido com a missão } \\
\text { de manter os vínculos e a conexão entre ambos. }\end{array}$ & $\begin{array}{l}\text { Queiroz; De } \\
\quad \text { Paula. }\end{array}$ & 2016 \\
\hline
\end{tabular}




\begin{tabular}{|c|c|c|c|}
\hline \multicolumn{4}{|c|}{ Quadro 1 - Artigos da amostra da pesquisa (continuação) } \\
\hline Título do Artigo & Objetivo do Artigo & Autores & Ano \\
\hline $\begin{array}{l}\text { CRM Framework for } \\
\text { Higher Education in } \\
\text { Mauritius }\end{array}$ & $\begin{array}{l}\text { Propor uma estrutura conceitual de CRM que pode } \\
\text { ser usada por universidades públicas nas Ilhas } \\
\text { Maurício, a fim de gerenciar e qualificar o } \\
\text { relacionamento entre essas instituições e seus alunos. }\end{array}$ & $\begin{array}{l}\text { Roopchund.; } \\
\text { Alsaid. }\end{array}$ & 2017 \\
\hline $\begin{array}{l}\text { Sticking with your } \\
\text { university: the importance } \\
\text { of satisfaction, trust, } \\
\text { image, and shared values }\end{array}$ & $\begin{array}{l}\text { Testar um modelo derivado de uma perspectiva de } \\
\text { marketing de relacionamento para investigar os } \\
\text { papéis de quatro variáveis (imagem de marca, } \\
\text { confiança, satisfação e valores compartilhados) na } \\
\text { explicação direta e indireta da lealdade de ex-alunos. }\end{array}$ & $\begin{array}{l}\text { Schlesinger; } \\
\text { Cervera; } \\
\text { Pérez- } \\
\text { Cabañero. }\end{array}$ & 2017 \\
\hline $\begin{array}{l}\text { The Role of Students and } \\
\text { Alumni in Relationship } \\
\text { Marketing: An Exploratory } \\
\text { Study at the Cape } \\
\text { Peninsula University of } \\
\text { Technology, South Africa }\end{array}$ & $\begin{array}{l}\text { Examinar as percepções de estudantes seniores (da } \\
\text { Universidade de Tecnologia da Península do Cabo) } \\
\text { em relação aos esforços da universidade para atrair, } \\
\text { manter e melhorar os relacionamentos contínuos com } \\
\text { alunos (e eventualmente com ex-alunos) }\end{array}$ & $\begin{array}{l}\text { A.A. } \\
\text { (Braa } \\
\text { m) } \\
\text { Rust }\end{array}$ & 2017 \\
\hline $\begin{array}{l}\text { An innovation model of } \\
\text { alumni relationship } \\
\text { management: Alumni } \\
\text { segmentation analysis }\end{array}$ & $\begin{array}{l}\text { Agrupar os egressos em segmentos para melhor } \\
\text { compreender as suas características, estilos de vida, } \\
\text { tipos de comportamento e interesses. }\end{array}$ & $\begin{array}{l}\text { Rattanametha } \\
\text { wong; } \\
\text { Sinthupinyo; } \\
\text { Chandrachai }\end{array}$ & 2018 \\
\hline $\begin{array}{l}\text { Determinants for the } \\
\text { commitment relationship } \\
\text { maintenance between the } \\
\text { alumni and the alma mater }\end{array}$ & $\begin{array}{l}\text { O principal objetivo deste estudo é compreender o } \\
\text { que impulsiona a pressuposição voluntária desses } \\
\text { papéis pelos egressos, o que significa abordar a } \\
\text { necessidade de encontrar os determinantes para a } \\
\text { manutenção do relacionamento de compromisso entre } \\
\text { esses egressos e sua instituição formadora. }\end{array}$ & $\begin{array}{l}\text { Pedro; Pereira; } \\
\text { Carrasqueira. }\end{array}$ & 2018 \\
\hline $\begin{array}{l}\text { Hotel school alumni's } \\
\text { intentions to use } \\
\text { communication channels-a } \\
\text { cross-generational } \\
\text { comparison study }\end{array}$ & $\begin{array}{l}\text { Investigar as perspectivas dos destinatários das } \\
\text { comunicações de marketing da associação de ex- } \\
\text { alunos, informando os canais que seus membros } \\
\text { pretendem usar, em diferentes gerações. }\end{array}$ & $\begin{array}{l}\text { Chen; } \\
\text { Murphy. }\end{array}$ & 2018 \\
\hline
\end{tabular}

Fonte: Elaborado pelos autores (2019).

Os resultados das análises da amostra de artigos apresentada no Quadro 1 são apresentados nas próximas subseções.

\subsection{Resultados de Métodos Empregados na Análise de Dados}

A categorização dos estudos selecionados foi realizada a partir da leitura do resumo e da seção de procedimentos metodológicos, buscando levantar as técnicas estatísticas empregadas pelos estudos sobre a temática. Após a identificação dos métodos quantitativos empregados, fez-se o registro dos mesmos para fins de apuração da frequência absoluta, frequência relativa e frequência relativa acumulada. 
Gráfico 1. Frequência de utilização dos métodos estatísticos quantitativos.

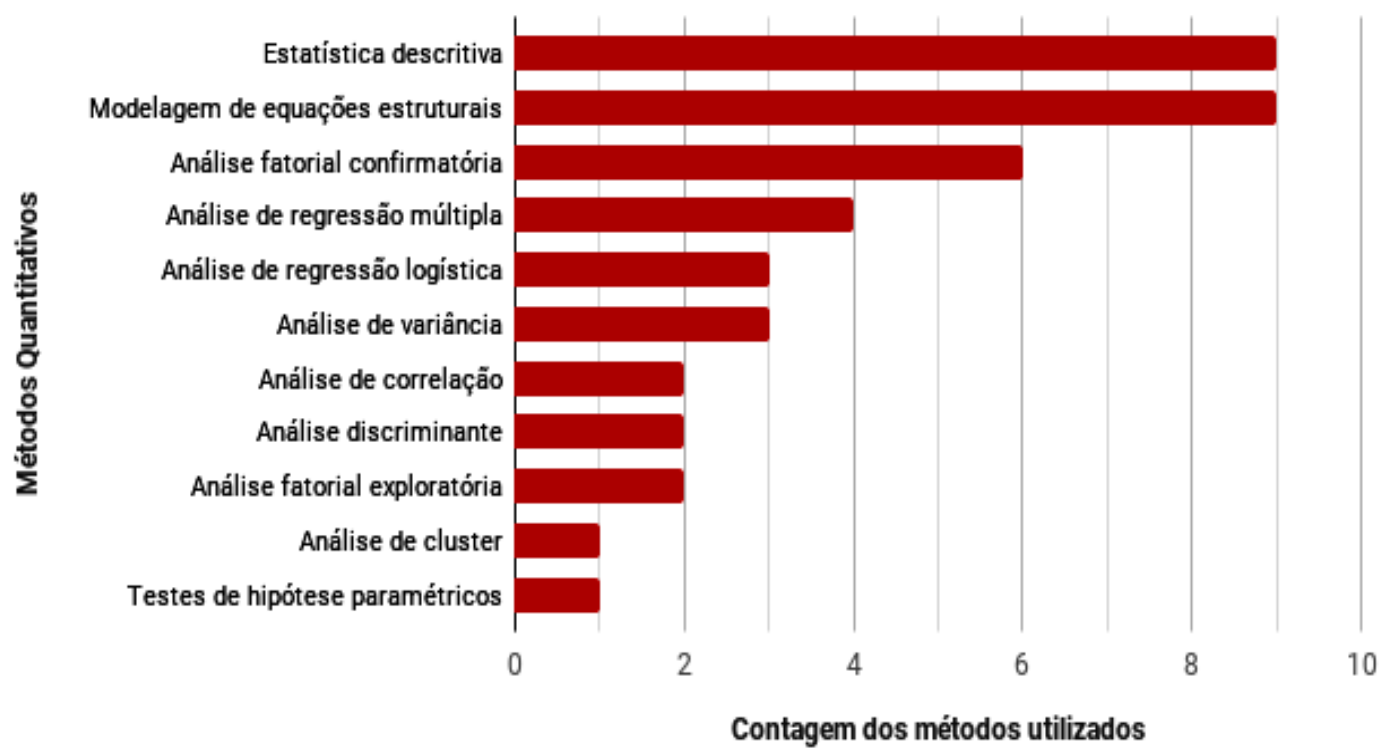

Fonte: Elaborado pelos autores (2019).

O Gráfico 01 apresenta os resultados de frequência de utilização dos métodos estatísticos pelos estudos na temática. Observa-se que a maior parte dos estudos publicados utiliza: técnicas de estatística descritiva (9 estudos), modelagem de equações estruturais (9) e análise fatorial confirmatória (6). Já as técnicas menos utilizadas são: análise de variância, análise de correlação, analise fatorial exploratória, análise de cluster e testes de hipóteses paramétricos. $\mathrm{Na}$ sequência foram identificados os esforços empreendidos na verificação dos pressupostos requeridos pelas técnicas de análise utilizadas.

\subsection{Resultados de Análise dos Pressupostos das Técnicas Utilizadas}

Conforme Gouvêa et al. (2012a), as técnicas estatísticas de análise possuem algumas suposições próprias (pressupostos ou premissas) que precisam ser previamente verificadas no conjunto de dados analisados, visando garantir que o algoritmo da técnica tenha o comportamento que se espera. Nas palavras de Hair Junior et al. (2009, p. 82) as "técnicas multivariadas e suas contrapartes univariadas são todas baseadas em um conjunto fundamental de suposições representando as exigências da teoria estatística inerente".

Nas pesquisas que compõem o portfólio bibliográfico deste estudo há 17 estudos, ou seja, a maioria deles (85\%), que mesmo apresentando a técnica de análise utilizada na seção metodologia, não evidenciam a verificação dos pressupostos requeridos. Isso não significa que esses pressupostos não tenham sido levados em consideração na elaboração do trabalho, mas que não constam evidências sobre a sua verificação na escrita do artigo. Observou-se, também, que quatro dos trabalhos analisados citaram os pressupostos adjacentes à técnica estatística selecionada, mas não apresentaram evidências sobre os testes realizados (nem seus resultados).

No estudo de Walesska Schlesinger, Cervera Taulet, Iniesta Bonillo, \& Sánchez Fernández (2014), a normalidade multivariada, um dos pressupostos das técnicas de modelagem de equações estruturais e da análise fatorial, foi verificada antes da análise dos dados. Essas técnicas possuam vários outros pressupostos a serem verificados, mas a normalidade multivariada é uma condição fundamental para aplicação dessas técnicas; assim 
como o uso de variáveis provenientes de escala métrica. Já o estudo de McDearmon (2013), que utilizou técnicas de estatística descritiva e de regressão logística, cita que fez a verificação da multicolinearidade dos dados e do tamanho da amostra. Os resultados indicam que não houve problemas de multicolinearidade, já que todos os valores do fator de inflação da variância foram relativamente baixos.

Conforme Gouvêa et al. (2012a), os efeitos da multicolinearidade dos dados podem ser classificados em termos de explicação e estimação. Na explicação é mais complicado determinar a contribuição de cada variável independente, pois os efeitos das variáveis independentes estão misturados, pois é difícil o processo de separação de efeitos individuais de cada variável independente sobre a variável dependente. Na estimação, os graus elevados de multicolinearidade podem fazer com que os coeficientes das variáveis independentes sejam incorretamente estimados, apresentando, inclusive, sinais errados.

Por fim, outro estudo que referenciou a verificação de pressupostos estatísticos é o trabalho de Torres e Brasil (2009), que analisou o tamanho da amostra, a ausência de outliers, a normalidade dos dados e a homoscedasticidade; tais premissas são requeridas na utilização da modelagem de equações estruturais. $\mathrm{O}$ critério utilizado para dimensionar o tamanho da amostra foi o de verificar se a quantidade de respondentes seria suficientemente para permitir o uso da técnica. Segundo Hair Junior et al. (2005), nessas situações o tamanho da amostra deve ter ao mínimo de cinco a dez respondentes por parâmetro estimado no modelo proposto.

Com relação aos outliers, ou observações atípicas, Hair Junior et al. (2009) afirmaram que estes podem exercer um efeito sensível sobre qualquer tipo de análise empírica. Portanto, precisam ser avaliados na perspectiva do quão são representativos na população. Assim, o pesquisador precisaria verificar se o valor marginal deve ser mantido ou eliminado, no caso de influência indevida sobre os resultados. Sobre a homoscedasticidade, Gouvêa et al. (2012a) ressaltaram que se trata de uma propriedade fundamental, pois a sua ausência pode invalidar toda a análise estatística. Quando a homoscedasticidade não é atendida, podem ser observadas melhores previsões em alguns níveis das variáveis independentes do que em outros.

\subsection{Resultados de Variáveis Analisadas}

O Gráfico 02 apresenta os resultados das variáveis (ou constructos) analisados nos estudos sobre marketing de relacionamento com egressos. Tais variáveis são: imagem da universidade, satisfação, comportamento colaborativo, lealdade, identificação egressouniversidade, qualidade da interação aluno-professor, reputação, comprometimento, confiança, valores compartilhados, expectativas do cliente, comunicação, afeição/apego emocional, relacionamento egresso-universidade, suporte acadêmico, suporte administrativo, acessibilidade, identificação e envolvimento/vínculo acadêmico. Todas essas variáveis estão presentes na literatura de marketing de relacionamento. 
Gráfico 2. Variáveis utilizadas nos estudos.

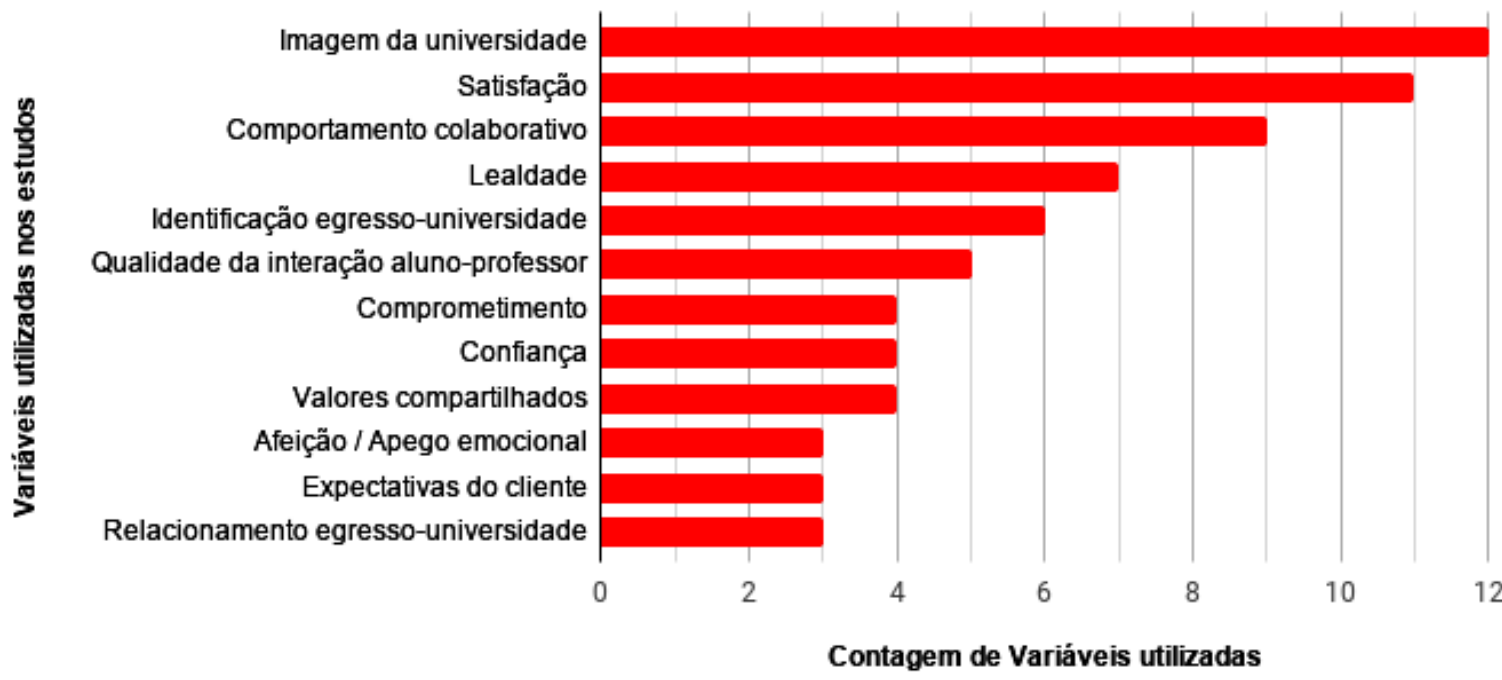

Fonte: Elaborado pelos autores (2019).

Nota-se que as variáveis mais analisadas são imagem institucional, satisfação, comportamento colaborativo, lealdade, identificação egresso-universidade e qualidade da interação. Essas variáveis dialogam diretamente na literatura de marketing de relacionamento no contexto da instituição de ensino, e estão presentes nos trabalhos de Reinert e Reinert (2004) e Lordelo e Dazzani (2012), os quais relatam que a construção de valor, satisfação, fidelidade e a gestão do relacionamento com o cliente são conceitos importantes tanto para organizações empresariais como instituições de ensino.

As variáveis mais recorrentes podem ser justificadas em função do conceito de marketing de relacionamento, que trabalha estreitamente a definição e a relação entre esses conceitos, como comprometimento, relacionamento continuado, afeição, motivação, lealdade e confiança (Moorman, Zaltman, \& Deshpande, 1992; Morgan, \& Hunt, 1994; Berry, 1995; Voss, \& Voss, 1997; Gruen, Summers, \& Acito, 2000; Lousada, \& Martins, 2005; Palmatier, Dant, Grewal, \& Evans (2006); Shukla, Banerjee, \& Singh, 2016; Su, Swanson, Chinchanachokchai, Hsu, \& Chen (2016).

\subsection{Resultados de escalas de mensuração utilizadas}

Fenômenos podem ser medidos de diferentes formas e maneiras, devendo ser observados os procedimentos de análise e modelos teóricos selecionados (Cooper \& Schindler, 2016). Dentre os trabalhos que compõem a amostra desta pesquisa, 14 deles (70\%) informaram utilizar a escala de mensuração em formato Likert. Conforme Cooper e Schindler (2016), a escala Likert consiste em um conjunto de frases, onde cada respondente pode expressar o seu grau de concordância (ou discordância) com algum dos pontos que compõem a escala. Assim, a escala Likert pode ser utilizada com diferentes níveis de detalhamento, ou diferentes quantidades de pontos, e cuja precisão acerca das informações que o pesquisador conseguirá está diretamente relacionada com a quantidade de pontos utilizados (Hair Junioret al., 2005). O Quadro 02 apresenta a frequência dos tipos de escala encontradas nos estudos sobre a temática. Os resultados mostram a predominância das escalas de 11 e 5 pontos. 
Quadro 02. Frequência da quantidade de pontos nas escalas utilizadas

\begin{tabular}{|c|c|}
\hline $\mathbf{N}^{\mathbf{0}}$ DE PONTOS DA ESCALA & OCORR̂̂NCIA \\
\hline 11 pontos & 6 artigos \\
5 pontos & 5 artigos \\
6 pontos & 2 artigos \\
7 pontos & 2 artigos \\
\hline
\end{tabular}

Fonte: Elaborado pelo autor (2019).

Ainda sobre a escala de Likert, Malhotra (2012) afirmou que uma de suas características é a de possuir uma estrutura simples, que permite a fácil elaboração, aplicação e entendimento por parte dos pesquisados, além de propiciar o preenchimento de questionários sem a presença do pesquisador. De fato, a maioria dos instrumentos de coleta utilizados nos estudos sobre o tema (em 85\% deles), a coleta de dados ocorreu por meio do método questionários auto-administrado, ou seja, preenchidos pelos próprios respondentes, sem o apoio do pesquisador envolvido.

\subsection{Resultados da Composição do Instrumento de Coleta}

Ao se analisar os artigos que compõem o portfólio bibliográfico desta pesquisa, constatouse que a totalidade deles utilizou o questionário como instrumento de coleta. Hair Junior et al. (2005) definem o questionário como um instrumento cientificamente desenvolvido, que possibilita medir características de indivíduos, empresas, eventos e fenômenos. Difere do formulário de entrevista por possibilitar ser auto-administrado, dispensando a presença do entrevistador (Vergara, 2012).

Os estudos sobre o tema aplicaram questionários utilizando os seguintes principais meios: plataformas online (presente em nove trabalhos), telefone (sete trabalhos), formulário impresso pessoalmente (três trabalhos) e pelo correio (um trabalho publicado em 2001 e com uma taxa de retorno de 59\%). Sobre o processo de elaboração, do total de estudos analisados, nove relataram detalhes do processo de construção, descrevendo inclusive as etapas qualitativas iniciais e de adaptação da escala ao contexto da pesquisa. Dentre esses, sete evidenciaram a realização de pré-testes ou validação de face do questionário. Entretanto, constatou-se que nenhum dos artigos da amostra apresentam um detalhamento sobre a composição do questionário, como quantidade de questões e estrutura do questionário. É bastante usual o uso de questionários em pesquisas sobre satisfação como mostra a pesquisa de Altaf, Matos, Tavares, Santos Jr., \& Lima (2015) e Altaf, Zancaneli e Falco (2016).

Sobre as técnicas empregadas na avaliação da confiabilidade do instrumento, 14 estudos descrevem sobre o seu uso, citando ter utilizado do coeficiente do Alfa de Cronbach. Assim, há predominância no uso dessa técnica de análise na verificação da consistência interna das escalas utilizadas; isso encontra respaldo em Hair Junior et al. (2009), que afirmaram ser o Alfa de Cronbach a medida de avaliação da confiabilidade do instrumento de coleta mais utilizada na literatura de business e marketing.

\subsection{Resultados de Bibliografia de Suporte Utilizada}

Por fim, a coleta de dados buscou ainda identificar a bibliografia de suporte utilizada sobre métodos quantitativos nos estudos sobre a temática. Há uma variedade de referências utilizadas, e a maior frequência observada não atinge metade da amostra analisada. O Quadro 03 apresenta as bibliografias de suporte com mais ocorrência de citação. Os artigos do 
portfólio bibliográfico aqui analisado citaram 39 vezes as 21 obras identificadas como referência para as análises estatísticas realizadas, tendo em alguns casos bibliografia não ligada à literatura de Estatística. No entanto, quatro artigos (10,3\% deles) descrevem os procedimentos metodológicos utilizados, sem evidenciar qualquer referência no tratamento estatístico na análise de dados.

Quadro 03 - Bibliografias de suporte utilizadas nos estudos na temática

\begin{tabular}{|l|c|}
\hline \multicolumn{1}{|c|}{ BIBLIOGRAFIA } & CITAÇÕES \\
\hline $\begin{array}{l}\text { ANDERSON, J. C.; GERBING, D. W. Structural equation modeling in } \\
\text { practice: a review and recommended two-step approach. Psychological } \\
\text { Bulletin, [S. 1.], v. 103, n. 3, p. 411- 423, 1988. }\end{array}$ & 6 \\
\hline $\begin{array}{l}\text { BAGOZZI, R.; YI, Y. On the evaluation of structural of equation models. } \\
\text { Journal of the Academy of Marketing Science, 16(1), 74-94, 1988. }\end{array}$ & 5 \\
\hline $\begin{array}{l}\text { FORNELL, C.; LARCKER, D. F. Evaluating structural equation models with } \\
\text { unobservable variables and measurement error, Journal of Marketing } \\
\text { Research, 18 (1), 39-50, 1981. }\end{array}$ & 5 \\
\hline $\begin{array}{l}\text { HAIR JR, J.; ANDERSON, R.; TATHAM, R.; BLACK, W. Análisis } \\
\text { Multivariante, 5. ed. Espanha: Prentice Hall, 2000. }\end{array}$ & 2 \\
\hline NUNNALLY, J. C. Psychometric theory. New York: McGraw-Hill, 1979. & 2 \\
\hline
\end{tabular}

Fonte: Elaborado pelo autor (2019)

Cabe ressaltar que um dos trabalhos mais referenciados é o artigo "Structural equation modeling in practice: a review and recommended two-step approach" publicado em 1988, por James Anderson e David Gerbing, no American Psychological Association, Psychological Bulletin, com 33 mil citações no Google Acadêmico, no dia 01 de maio de 2019, e 14.912 citações no Web of Science na mesma data. Os outros dois trabalhos mais referenciados são: "On the evaluation of structural of equation models", publicado em 1988, por Richard P. Bagozzi e Youjae Yi, no Journal of the Academy of Marketing Science, com 20.589 citações no Google Acadêmico; e o artigo "Evaluating structural equation models with unobservable variables and measurement error", publicado em 1981 por Claes Fornelle David Larcker no Journal of Marketing Research, com 53.036 citações no Google Acadêmico e 24.984 citações no Web of Science.

Observa-se que os três trabalhos mais referenciados pelos estudos na temática são artigos com elevada quantidade de citações, e publicados em periódicos científicos há mais de três décadas; somente no quarto lugar é que aparece um livro propriamente de estatística, a obra de Joseph F. et al. "Análisis Multivariante", referenciada na sua $5^{\mathrm{a}}$ edição; que se encontra atualmente na $8^{\mathrm{a}}$ edição (2018). As demais 16 referências levantadas nos artigos receberam somente uma citação cada, sendo que entre estas está o artigo de Lee J. Cronbach, publicado em 1951, no qual o autor descreve sobre o coeficiente que levou o seu nome, e que estima a confiabilidade de uma escala de mensuração.

\section{Considerações Finais}

Este estudo buscou mapear o emprego dos métodos de pesquisa quantitativos nas publicações relacionadas ao tema marketing de relacionamento com egressos. Os resultados apontaram que os métodos de análise estatística mais utilizadas são técnicas de estatística descritiva, seguida de modelagem de equações estruturais e da análise fatorial confirmatória. Apontaram também que todos os estudos utilizaram o survey como estratégia de pesquisa, com a aplicação de questionários por meio de plataformas online, contato telefônico, envio 
por correios ou com aplicação presencial. Confirmou queo coeficiente do Alfa de Cronbach é o teste de consistência interna e de confiabilidade de escalas mais utilizado.

Com relação às escalas utilizadas, os resultados do estudo mostram que a escala Likert é a mais utilizada nos instrumentos de coleta de dados, principalmente as escalas de 11 pontos e de cinco pontos. Já as variáveis analisadas pelos estudos na temática foram: imagem da universidade, satisfação, comportamento colaborativo, lealdade, identificação egressouniversidade, qualidade da interação aluno-professor, reputação, comprometimento, confiança, valores compartilhados, expectativas do cliente, afeição/apego emocional, suporte administrativo, relacionamento egresso-universidade, suporte acadêmico, acessibilidade, identificação, comunicação e envolvimento/vínculo acadêmico.

Os resultados deste estudo apontam ainda que muitos artigos na temática não evidenciam, de forma acurada, os procedimentos metodológicos adotados, impedindo uma análise mais aprofundada das técnicas estatísticas adotadas, e que nenhum dos artigos apresenta um detalhamento sobre a composição do questionário aplicado. Isso precisaria ser melhor observado em publicações de estudos futuras na temática. A despeito de tal observação, esta pesquisa atendeu ao seu objetivo principal que é o de mapear as principais técnicas e métodos estatísticos adotados nos estudos publicados em formato de artigos e em periódicos científicos sobre o tema marketing de relacionamento com egressos.

Houve limitações no desenvolvimento desta pesquisa. O conjunto de bases de dados, diretórios e portais em que foram feitas as buscas, embora tenham sido escolhidos por serem tradicionalmente usados em pesquisas da área de Administração, não esgotam o conjunto de opções de buscas existente. Outra limitação é que embora as palavras-chave e as operações booleanas por si só sejam não-enviesadas, a escolha do conjunto de palavras-chave e proposição das operações booleanas contém a subjetividade da experiência dos pesquisadores. Por fim, a aplicação do filtro da leitura dos resumos e palavras-chave é inerentemente subjetiva e pode gerar resultados diferentes quando aplicados por diferentes pesquisadores.

Por fim, o presente estudo não pretendeu esgotar o assunto, mas contribuir para a disseminação dos modelos estatísticos de análise utilizados na temática e de incentivar a adoção de algumas técnicas de análise ainda pouco exploradas. Assim, recomenda-se também que futuros estudos na temática ampliem a fonte de dados analisados, abrangendo Anais de eventos, teses e dissertações.

\section{Referências}

Agresti, A. (2019). An introduction to categorical data analysis. ( $3^{a}$ ed.). New Jersey: John Wiley \& Sons.

Almeida, J. (2014). Como aumentar a probabilidade de aprovação de artigos em periódicos? Análise dos pareceres de avaliadores da Revista Brasileira de Contabilidade. Revista Brasileira de Contabilidade, 206, 13-25.

Altaf, J. G., Matos, D. M., Tavares, L. T., Santos Junior., O. A., \& Lima, R. I. N. (2015). Clínica Olhar: Análise da Satisfação dos Clientes em Relação ao Serviço Prestado. RASI, 1(1), 1-36. doi: 10.20401/rasi.1.1.19. 
Altaf, J. G., Zancanello, L. C., \& Falco, G. P. (2016). Aspectos Preponderantes para a Satisfação do Cliente: o Caso 25 de Março. RASI, 2(1), 67-86. doi: 10.20401/rasi.2.1.74.

Barbetta, P. (2017). Estatística aplicada às ciências sociais. ( $8^{\mathrm{a}}$ ed.). Florianópolis: UFSC.

Berry, L. (1995). Relationship marketing of services-growing interest, emerging perspectives. Journal of The Academy of Marketing Science, 23(4), 236-245. doi: $10.1177 / 009207039502300402$.

Chen, P., \& Popovich, P. (2002). Correlation: parametric and nonparametric measures. London: Sage.

Cooper, D., \& Schindler, P. (2016). Métodos de Pesquisa em Administração. (12a . ed.). Porto Alegre: AMGH.

Dallabona, L., Nascimento, S., \& Hein, N. (2010). Métodos estatísticos mais recorrentes nas dissertações do programa de pós-graduação em Ciências Contábeis da FURB. Revista de Contabilidade da UFBA, 4(1), 56-70.

Espejo, M., Azevedo, S., Trombelli, R., \& Voese, S. (2013). O mercado acadêmico contábil brasileiro: uma análise do cenário a partir das práticas de publicação e avaliação por pares. Revista Universo Contábil, 9(4), 06-28. doi: 10.4270/RUC.2013428.

Falaster, C., Ferreira, M. P., \& Canela, R. (2016). Motivos de rejeição dos artigos nos periódicos de administração. Organizações \& Sociedade, 23(77), 285-306. doi: 10.1590/19849230776.

Fávero, L. P. L., \& Belfiore, P. (2017). Manual de análise de dados: estatística e modelagem multivariada com Excel, SPSS e Stata. Rio de Janeiro: Elsevier.

Fávero, L. P., Belfiori, P., Silva, F. L., \& Chan, B. L. (2009). Análise de dados: modelagem multivariada para tomada de decisões. Rio de Janeiro: Elsevier.

Ferreira, M. P., \& Falaster, C. (2016). Uma Análise Comparativa dos Fatores de Rejeição nos Periódicos de Diferentes Estratos de Administração. Revista de Administração Contemporânea, 20(4), 412-433. doi: 10.1590/1982-7849rac2016140144.

Fiates, G. G. S., Serra, F. A. R., \& Martins, C. (2014). A aptidão dos pesquisadores brasileiros pertencentes aos programas de pós-graduação stricto sensu em Administração para pesquisas quantitativas. Revista de Administração, 49(2), 384-398. doi: 10.5700/rausp1153.

Field, A. (2009). Descobrindo a estatística usando o SPSS. (2a ed.).Porto Alegre: Artmed.

Gouvêa, M. A., Prearo, L. C., \& Romeiro, M. C. (2010). Abordagem exploratória do emprego das técnicas de análise de regressão e análise conjunta em estudos do comportamento do consumidor. Revista Brasileira de Gestão de Negócios, 12(36), 253-270. doi: 10.7819/rbgn.v12i36.661. 
Gouvêa, M., Prearo, L., \& Romeiro, M. (2012a). Avaliação da adequação de aplicação de técnicas multivariadas em estudos do comportamento do consumidor em teses e dissertações de duas instituições de ensino superior. Revista de Administração (São Paulo. Online), 47(2), 338-355. doi: 10.1590/S0080-21072012000200013.

Gouvêa, M., Prearo, L., \& Romeiro, M. (2012b). Avaliação da aplicação de técnicas multivariadas de interdependência em teses e dissertações de algumas Instituições de Ensino Superior. FACEF Pesquisa: Desenvolvimento e Gestão, 15(1), 107-124.

Gouvea, M., Prearo, L., \& Romeiro, M. (2011). Avaliação do emprego da técnica de análise multivariada de variância em teses e dissertações de algumas instituições de ensino superior. Estudos do CEPE, 34, 69-97. doi:10.17058/cepe.v0i34.2149.

Gouvêa, M., Prearo, L., \& Romeiro, M. (2013). Avaliação do emprego das técnicas de Análise de correspondência e análise de agrupamentos em teses e dissertações de algumas instituições de ensino superior. Revista de Ciências da Administração (CAD/UFSC), 15(35), 52-67. doi: 2175-8077.2013v15n35p52.

Gray, D. (2012). Pesquisa no mundo real. (2ª . ed.). Porto Alegre: Penso.

Gruen, T., Summers, J., \& Acito, F. (2000). Relationship marketing activities, commitment, and membership behaviors in professional associations. Journal of Marketing, 64(3), 34-49.

Hair Junior., J. F., Black, W. C., Babin, B. J., Anderson, R. E., \& Tatham, R, L. (2009). Análise multivariada de dados. ( $6^{\mathrm{a}}$ ed.). Porto Alegre: Bookman.

Hair Junior., J. F., Babin, B., Money, A. H., \& Samouel, P. (2005). Fundamentos de métodos de pesquisa em administração. Porto Alegre: Bookman.

Hosser, C., Cruz, A. P. C., \& Quintana, A. C.(2018). Mapeamento dos métodos quantitativos utilizados no congresso Anpcont (2007-2015). Revista de Contabilidade da UFBA, 12(3), 153-174. doi: 10.9771/rc-ufba.v12i3.26342.

Lana, J., Partyka, R. B., Alberton, A., \& Marcon, R. (2018). Caso para ensino: o processo de escolhas metodológicas em uma abordagem quantitativa. Revista de Contabilidade e Organizações, 12(1), 1-11. doi: 10.11606/issn.1982-6486.rco.2018.148286.

Larson, R., \& Farber, L. (2010). Estatística aplicada. (4ª ed.). São Paulo: Pearson.

Lattin, J., Carroll, J., \& Green, P. (2011). Análise de dados multivariados. São Paulo: Cengage Learning.

Levin, J., Fox, J., \& Forde, D. (2012). Estatística para ciências humanas. (11 ${ }^{\mathrm{a}}$. ed.). São Paulo: Pearson.

Lordelo, J., \& Dazzani, M. (2012). Estudos com estudantes egressos: concepções e possibilidades metodológicas na avaliação de programas. Salvador: EDUFBA. 
Lousada, A. C. Z., \& Martins, G. A. (2005). Egressos como fonte de informação à gestão dos cursos de Ciências Contábeis. Revista Contabilidade \& Finanças, 16(37), 73-84. doi: 10.1590/S1519-70772005000100006.

Malhotra, N. (2012). Pesquisa de marketing: uma orientação aplicada. (6 ${ }^{\text {a }}$ ed.). Porto Alegre: Bookman.

Mcdearmon, J. (2013). Hail to thee, our alma mater: alumni role identity and the relationship to institutional support behaviors. Research in Higher Education, 54(3), 283-302. doi: 10.1007/s11162-012-9271-6.

Moorman, C., Zaltman, G., \& Deshpande, R. (1992). Relationships between providers and users of market research: the dynamics of trust within and between organizations. Journal of Marketing Research, 29(3), 314-328. doi: 10.1177/002224379202900303.

Morgan, R., \& Hunt, S. (1994). The commitment trust theory of relationship marketing. Journal of Marketing, 58(3), 20-38. doi: 10.2307/1252308.

Palmatier, R. W., Dant, R. P., Grewal, D., \& Evans, K. R. (2006). Factors Influencing the Effectiveness of Relationship Marketing: A Meta-Analysis. Journal of Marketing, 70(4), 136153. doi: 10.1509/jmkg.70.4.136.

Prearo, L., Gouvêa, M., \& Monari, C. (2010). Avaliação do emprego da técnica de análise discriminante em teses e dissertações de algumas instituições de ensino superior. Revista de Administração FACES Journal, 9(1), 129-147. doi: 1984-6975FACES2010V9N1ART177.

Prearo, L., Gouvêa, M., \& Monari, C. (2009). Avaliação do emprego da técnica de análise de regressão logística em teses e dissertações de algumas instituições de ensino superior. Semina. Ciências Sociais e Humanas (Online), 30(2), 37-54, 2009. doi: 10.5433/16790383.2009v30n2p123.

Prearo, L., Gouvêa, M., Monari, C., \& Romeiro, M. (2011). Avaliação do emprego da técnica de Análise fatorial em teses e dissertações de algumas instituições de ensino superior. REGE. Revista de Gestão USP, 18(4), 621-638. doi: 10.5700/rege441.

Prearo, L., Gouvêa, M., \& Romeiro, M. (2012). Avaliação da adequação de aplicação de técnicas multivariadas de dependência em teses e dissertações de algumas instituições de ensino superior. Ensaios FEE (Online), 33(1), 261-290.

Prearo, L., Gouvêa, M., \& Romeiro, M. (2011a). Avaliação do emprego das técnicas de análise de regressão e correlação canônica em teses e dissertações de algumas instituições de ensino superior. Revista Ciências Administrativas (UNIFOR), 17(3), 691-727.10.5020/23180722.17.3.\%25p.

Prearo, L., Gouvêa, M., \& Romeiro, M. (2011b). Avaliação do emprego da técnica de Modelagem de equações estruturais em teses e dissertações de universidades públicas de alta performance. Revista da FAE, 14(2), 80-99. 
Reinert, J. N., \& Reinert, C. (2004). Estudante não é cliente: É parceiro. Artigo apresentado no IV Colóquio Internacional de Gestão Universitário na América do Sul, Florianópolis.

Roses, C., \& Leis, R. (2002). Um estudo das condições socioeconômicas de municípios gaúchos através da análise de cluster. Revista Administração Online, 3(3), n.p.

Sampieri, R., Collado, C., \& Lúcio, M. (2013). Metodologia de pesquisa. (5 ed.). Porto Alegre: Penso.

Schlesinger, M. W., Taulet, A. C., Bonillo, M. Á. I., \& Fernández, R. (2014). Un enfoque de marketing de relaciones a la educación como un servicio: aplicación a la Universidad de Valencia. Innovar, 24(53), 113-125. doi: 10.15446/innovar.v24n53.43919.

Silva, A. C., Wanderley, C. A. N., \& Santos, R. D. (2010). Utilização de ferramentas estatísticas em artigos sobre contabilidade financeira - um estudo quantitativo em três congressos realizados no país. Revista Contemporânea de Contabilidade, 7(14), 11-28. doi: 10.5007/2175-8069.2010v7n14p11.

Soares, S., Picolli, I., \& Casagrande, J. (2018). Pesquisa bibliográfica, pesquisa bibliométrica, artigo de revisão e ensaio teórico em Administração e Contabilidade. Administração: Ensino e Pesquisa, 19(2), 308-339. doi: 10.13058/raep.2018.v19n2.970.

Steiner, M. (1995). Uma metodologia para o reconhecimento de padrões multivariados com resposta dicotômica.(Tese de doutorado, Universidade Federal de Santa Catarina). Recuperado de: https://repositorio.ufsc.br/xmlui/handle/123456789/157952.

Su, L., Swanson, S. R., Chinchanachokchai, S., Hsu, M. K.,\& Chen, X. (2016). Reputation and intentions: The role of satisfaction, identification, and commitment. Journal of Business Research, 69(9), 3261-3269. doi: 10.1016/j.jbusres.2016.02.023.

Voss, G., \& Voss, Z. (1997). Implementing a relationship marketing program: A case study and managerial implications. Journal of Services Marketing, 11(4), 278-298. doi:10.1108/08876049710171731. 